\title{
RUNX1/RUNX1T1 Fusion Positive
}

National Cancer Institute

\section{Source}

National Cancer Institute. RUNX1/RUNX1T1 Fusion Positive. NCI Thesaurus. Code C128837.

Indicates that expression of the RUNX1/RUNXT 1 fusion has been detected in a sample. 